\title{
An Explanation for the Shape of the Human Penis
}

\author{
Edwin A. Bowman
}

Published online: 23 October 2009

(C) Springer Science+Business Media, LLC 2009

The present form of the human penis, reached over several million years of evolution, was orchestrated by the vagina of the human female. The multifunctional vagina serves as a birth canal, a component of the structural support for the internal pelvic organs, and as a coital organ. In this last function, it has had an evolutionary effect in shaping male sexual anatomy. Hominid fossils so far discovered show that the female pelvis evolved to accommodate the increasing size of the brain of the human infant. Larger cranial capacity necessitated a larger birth canal and, as a result of sexual selection, an increase in the size of the penis. Compared to that of the other great apes, the human penis is considerably larger, and evidence suggests that its unique configuration may also be a result of vaginal influence.

It has been proposed that the shape of the glans with its distinctive corona facilitates the scooping out of previously deposited semen, enabling the subsequent deposition of other genetic material. This is not a likely occurrence. The healthy vagina has a low $\mathrm{pH}$ to protect it from invading organisms. This intense acidity will kill all sperm not promptly reaching the safety of the cervical mucus. I suggest the evolutionary function of the glans and its corona is not to scoop out semen but vaginal secretions, which are then absorbed by the encompassing foreskin. The mucous membrane of the foreskin adjacent to the glans is highly absorbent, as evidenced by the increased susceptibility of the uncircumcised male to HIV. It has been suggested that there may be a glans protecting feature of the foreskin, but it is entirely possible that its primary function is to absorb behavior modifying chemical messengers from the female. Numerous studies have shown the vaginal mucosa to be secretory. In the primate rear-approach coital position, the corona rubs against the anatomically posterior wall of the vagina, the most dependent surface when the woman is standing, and therefore the place more likely to collect vaginal secretions. The deepest end of the posterior vagina is the cul de sac, which commonly contains a small amount of fluid. An analytical study of the vaginal secretions of an ovulating female for neuropeptides, such as pitocin and vasopressin, known to enhance pair bonding and protective behavior in the male, could be revealing. Sexual intercourse may be a medium of communication between humans older than language itself.
E. A. Bowman ( $\square)$

515 Holly Point Road, Freeport, FL 32439, USA

e-mail: eabow@cox.net 